\title{
Two-Nucleon Knockout Spectroscopy at the Limits of Nuclear Stability
}

\author{
E. C. Simpson, ${ }^{1}$ J. A. Tostevin, ${ }^{1}$ D. Bazin, ${ }^{2}$ B. A. Brown, ${ }^{2,3}$ and A. Gade ${ }^{2,3}$ \\ ${ }^{1}$ Department of Physics, Faculty of Engineering and Physical Sciences, University of Surrey, \\ Guildford, Surrey GU2 7XH, United Kingdom \\ ${ }^{2}$ National Superconducting Cyclotron Laboratory, Michigan State University, East Lansing, Michigan 48824, USA \\ ${ }^{3}$ Department of Physics and Astronomy, Michigan State University, East Lansing, Michigan 48824, USA
}

(Received 9 December 2008; published 3 April 2009)

\begin{abstract}
Sudden single-nucleon removal reactions from fast radioactive beams are now key to studies of the structure of rare isotopes. The sensitivity of the heavy residue's parallel momentum distribution to the orbital angular momentum of the removed nucleon is a crucial feature with a high spectroscopic value. Twonucleon removal reactions provide experimental reach toward the rarest nuclear species. We show that the residue parallel momentum distributions in these reactions offer a clear spectroscopic signal of the angular momentum of the pair of nucleons removed, and thus of the residue final state spins and spectroscopy. Our formalism is applied successfully to new final-state-inclusive measurements of like-nucleon pair removal reactions to states in neutron-rich ${ }^{36} \mathrm{Mg}$ and neutron-deficient ${ }^{20} \mathrm{Mg}$. We also confront a new final-stateexclusive decomposition of two-proton knockout data to states in neutron-rich ${ }^{26} \mathrm{Ne}$.
\end{abstract}

DOI: 10.1103/PhysRevLett.102.132502

PACS numbers: 23.20.Lv, 21.60.Cs, 24.50.+g, 27.30.+t

Intermediate-energy one-nucleon knockout reactions from rare-isotope beams are now key to our obtaining spectroscopic information on the dominant proton and neutron single-particle structures near the Fermi surfaces of short-lived isotopes far from the valley of $\beta$ stability $[1,2]$. The potency of the method, aside from its high experimental efficiency, is the combination of measurements of cross sections and their distribution with the parallel momentum of the projectilelike residues that derive information on the spectroscopic strength and orbital angular momentum of the removed nucleon, respectively. Analyses have been used to deduce quantitative singleparticle spectroscopy in many instances, e.g., [3-6], and contributed to our understanding of the evolution of shell structures in nuclei with the most extreme $N: Z$ ratios. Recent examples include [7-10].

We consider analogous two-nucleon removal reactions, events in which intermediate-energy, mass $A$ projectiles undergo grazing collisions with a light nuclear target, resulting in the sudden removal of two like nucleons. The reactions of interest involve the removal of two protons from neutron-rich and two neutrons from neutron-deficient projectiles. In both cases the removed nucleons are strongly bound. We describe measurements where only the mass $A-2$ projectile residues (denoted $c$ ) are detected. Ideally, the energy of the final states of the residues are also identified by measurement of their in-flight decay $\gamma$ rays.

Key to physical interpretation is that, in the sudden approximation, the sum, $\vec{\kappa}_{1}+\vec{\kappa}_{2}$, of the momenta of the removed nucleons in the projectile rest frame is probed by the laboratory momenta of the projectile $\vec{K}_{A}$ and the residue $\vec{K}_{c}$ measured in two-nucleon removal events,

$$
\vec{\kappa}_{1}+\vec{\kappa}_{2}=-\vec{\kappa}_{c}=\frac{[A-2]}{A} \vec{K}_{A}-\vec{K}_{c},
$$

shown schematically in Fig. 1. Experiments determine the residue momentum component relative to a single direction. While early work on halo nuclei [11] looked at the component transverse to the beam direction, the distributions with respect to the parallel component are now preferred, these being less affected by (the principally transverse) Coulomb and diffractive scattering details within the reaction mechanisms. Henceforth we consider only the components in Eq. (1) parallel to the incident beam direction $\vec{K}_{A}$ (the $z$ axis).

This work advances significantly the approach developed $[12,13]$ for calculations of two-nucleon removal cross section yields to the distribution of cross section with the residue parallel momentum component. For clarity, we express the formalism with reference to the inelastic twonucleon removal (or stripping) mechanism. We do so as the calculated distributions of the stripping and the stripping plus diffraction mechanisms [13] are found to be essen-

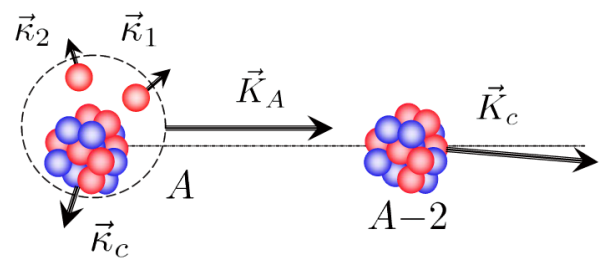

FIG. 1 (color online). Schematic of the momenta $\vec{\kappa}_{i}, i=1,2$, $c$, of the removed nucleons and the residue in the projectile rest frame, and of the projectile and the residue momenta $\vec{K}_{A}$ and $\vec{K}_{c}$ in the laboratory frame, that enter into the sudden approximation momentum relationship of Eq. (1). 
tially identical. The calculations presented include both of these mechanisms. The full details will be presented elsewhere [14].

Fundamentally, sudden two-nucleon removal reactions are highly constrained geometrically by the requirement that two nucleons undergo violent or absorptive interactions with the light target but the residue does not. At intermediate energy, the highly absorptive nature of the elastic $S$ matrices $\mathcal{S}_{i}, i=1,2, c$, of the nucleons and the residue with the target, results in very strong near-surface localization, as is represented schematically in Fig. 2. These geometry considerations are encoded in the calculations through the eikonal two-nucleon stripping operator

$$
\mathcal{A}(c, 1,2)=\left|\mathcal{S}_{c}\right|^{2}\left(1-\left|\mathcal{S}_{1}\right|^{2}\right)\left(1-\left|\mathcal{S}_{2}\right|^{2}\right),
$$

the joint probability that, in a collision at a projectile impact parameter $b$, the two nucleons are removed from their elastic channel but the residue survives.

Assuming an even-even spin-zero projectile, appropriate to our ${ }^{38} \mathrm{Si}$ and ${ }^{22,28} \mathrm{Mg}$ cases, the shell model two-nucleon overlap sampled in a reaction, with total angular momentum $J M$ is

$$
\Psi_{J M}=\sum_{j_{1} j_{2}}(-1)^{J-M} \frac{C\left(j_{1} j_{2} J\right)}{\sqrt{2 J+1}}\left[\overline{\phi_{j_{1}} \otimes \phi_{j_{2}}}\right]_{J M}
$$

with $C\left(j_{1} j_{2} J\right)$ the two-nucleon amplitudes (TNA) for the active two-nucleon configurations. In the sudden plus eikonal approximation, the two-nucleon stripping cross section to a residue final state $J$ can be expressed in terms of the joint position probabilities of the pair of nucleons in the plane normal to the beam direction. Explicitly, the $P_{J}\left(\vec{s}_{1}, \vec{s}_{2}\right)=\sum_{M} \int d z_{1} \int d z_{2}\left\langle\left|\Psi_{J M}\right|^{2}\right\rangle_{\mathrm{sp}} \quad$ quantify the removed-nucleons' position correlations after integration along the beam direction (the $z$ axis) and summation over intrinsic spin projections, the latter due to our lack of spin selectivity. At each impact parameter $b$, the structure and geometrical factors, $P_{J}$ and $\mathcal{A}$, respectively, then deter-

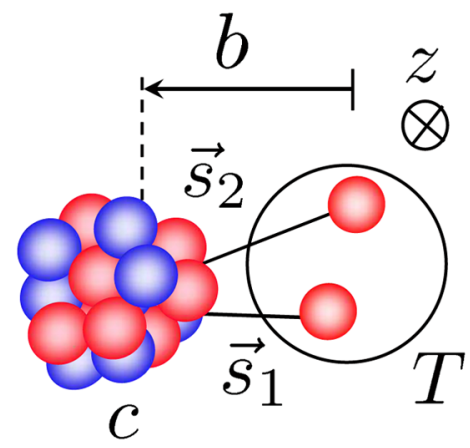

FIG. 2 (color online). Representation of the cylindrical volume, near the projectile surface, where the two-nucleon overlap function is sampled by the target $T$ at impact parameter $b$. This extends in the beam direction, the $z$ axis. $\vec{s}_{i}$ are the components of the position vectors of the removed nucleons relative to the residue in the plane normal to the beam. mine the exclusive stripping cross sections

$$
\sigma_{\text {str }}^{(J)}=\int d \vec{b} \int d \vec{s}_{1} \int d \vec{s}_{2} P_{J}\left(\vec{s}_{1}, \vec{s}_{2}\right) \mathcal{A}(c, 1,2) .
$$

Our emphasis here is the residue parallel momentum distribution. While the spatial reaction sampling $\mathcal{A}(c, 1,2)$ is unchanged, we now require the nucleons' joint position probabilities for each total $z$ component of their momentum. This is the $z$ component of the residue momentum, since $\kappa_{c}=-\left[\kappa_{1}+\kappa_{2}\right]$ in the projectile rest frame: see Fig. 1 and Eq. (1). The differential joint probability distribution is

$$
\begin{aligned}
\mathcal{P}_{J}\left(\vec{s}_{1}, \vec{s}_{2}, \kappa_{c}\right)= & \sum_{M}\left\langle\int d \kappa_{1} \int d \kappa_{2} \frac{\delta\left(\kappa_{c}+\kappa_{1}+\kappa_{2}\right)}{(2 \pi)^{2}}\right. \\
& \left.\times\left|\int d z_{1} \int d z_{2} e^{i \kappa_{1} z_{1}} e^{i \kappa_{2} z_{2}} \Psi_{J M}\right|^{2}\right\rangle_{\mathrm{sp}},
\end{aligned}
$$

and the final-state-exclusive parallel momentum distribution is

$$
\frac{d \sigma_{\mathrm{str}}^{(J)}}{d \kappa_{c}}=\int d \vec{b} \int d \vec{s}_{1} \int d \vec{s}_{2} \mathcal{P}_{J}\left(\vec{s}_{1}, \vec{s}_{2}, \kappa_{c}\right) \mathcal{A}(c, 1,2)
$$

Inspection of Eq. (6) shows that if the reaction is strongly localized with impact parameter $b$, as is the case for removal of strongly-bound nucleons, and if the $\mathcal{P}_{J}$ do not change rapidly with the nucleon positions over the (relatively small) range of $\vec{s}_{i}$ sampled by the target, then the shapes of the $d \sigma_{\text {str }}^{(J)} / d \kappa_{c}$ distributions can be estimated from those of the $\mathcal{P}_{J}$ at fixed nucleon positions $\vec{s}_{i}$ near the projectile surface. This was the basis of a short earlier communication [15]. The complete calculations of Eq. (6) of this work confirm that these earlier estimates provide a

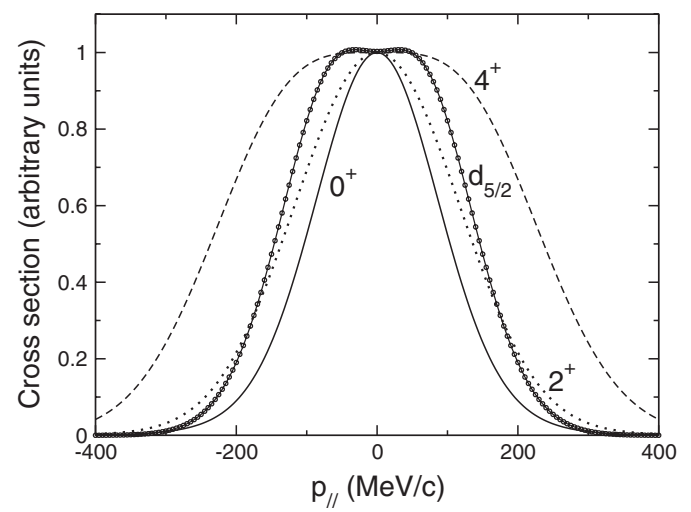

FIG. 3. Momentum distributions $d \sigma^{(J)} / d \kappa_{c}$ of the $J^{\pi}=0^{+}$, $2^{+}, 4^{+}$states in ${ }^{26} \mathrm{Ne}$ for pure $\pi\left[1 d_{5 / 2}\right]^{2}$ two-proton removal from ${ }^{28} \mathrm{Mg}$ at $82 \mathrm{MeV} / \mathrm{u}$. The final states are assumed to be degenerate in energy so as to clarify the effects of angular momentum coupling. The distribution for one-proton removal is also shown (solid curve and symbols), clarifying the narrow width of the $0^{+}$, two-proton distribution. All curves have been scaled to the same peak value. 
rather accurate description of these shapes [14]. This shows, fundamentally, that the shapes of the distributions are very robust and result from the geometrical selectivity of the reaction. It is for this reason that the stripping and the stripping plus diffraction mechanisms lead to very similar distributions.

A significant result, for spectroscopy, is the strong $J$ dependence of both the shapes and widths of the calculated distributions. These are shown in Fig. 3 for $J^{\pi}=0^{+}, 2^{+}$ and $4^{+}$final state transitions in ${ }^{26} \mathrm{Ne}$, when assuming a pure $\pi\left[1 d_{5 / 2}\right]^{2}$ two-proton removal from ${ }^{28} \mathrm{Mg}$, at $82 \mathrm{MeV} / \mathrm{u}$. The inputs to these and the following theoretical calculations were as follows. The eikonal $S$ matrices $\mathcal{S}_{i}\left(b_{i}\right)$ were calculated as described in [13], the neutron and proton point particle densities in each residue being taken from spherical Skyrme (SkX) Hartree-Fock (HF) calculations [16]. All the reactions considered were on a ${ }^{9} \mathrm{Be}$ target. The ${ }^{9} \mathrm{Be}$ was assumed to have a Gaussian density with root-mean-squared (rms) radius $2.36 \mathrm{fm}$. The nucleon single-particle wave functions were calculated in WoodsSaxon potentials of a fixed diffuseness $a=0.7 \mathrm{fm}$ and with radius parameters $r_{0}$ that reproduce the rms radii of the active orbits given by the HF calculation: see [17]. A spin-orbit potential of strength of $V_{\mathrm{so}}=6 \mathrm{MeV}$ was used and the depths of the central potentials were chosen to reproduce the separation energies appropriate to the ground and excited-state transitions. The two-nucleon separation energy was shared equally between the two removed nucleons.

For the specific applications to ${ }^{22,28} \mathrm{Mg}$ and ${ }^{38} \mathrm{Si}$ secondary beams the ground state two-nucleon configurations and their TNA, entering Eq. (3), were as used in the analyses of the cross section data in Refs. [18,12,19]. We consider quantitatively the residue momentum distributions of the two-like-nucleon knockout reactions ${ }^{9} \mathrm{Be}\left({ }^{28} \mathrm{Mg},{ }^{26} \mathrm{Ne}\right) X$, ${ }^{9} \mathrm{Be}\left({ }^{22} \mathrm{Mg},{ }^{20} \mathrm{Mg}\right) X$, and ${ }^{9} \mathrm{Be}\left({ }^{38} \mathrm{Si},{ }^{36} \mathrm{Mg}\right) X$ at midtarget energies of 82,75 , and $83 \mathrm{MeV} / \mathrm{u}$. All measurements were made at the National Superconducting Cyclotron Laboratory at Michigan State University using event-byevent trajectory reconstruction with the large-acceptance S800 spectrograph [20]. The neutron-rich and neutrondeficient projectiles were produced by projectile fragmentation. The particle identification in the entrance and exit channels, the details of the associated experimental setups and the magnitudes of the measured and theoretical cross sections are described in $[21,18,19]$. The momentum widths of the incoming ${ }^{22,28} \mathrm{Mg}$ and ${ }^{38} \mathrm{Si}$ beams were restricted to $0.5 \%, 0.5 \%$, and $1.66 \%$, respectively. The momentum profiles of the unreacted beams passing through the target were measured in the spectrograph and these experimental resolutions are taken into account in the comparisons with the calculated distributions. The location of the reaction vertex inside the target is also unknown, which introduces an additional momentum spread due to the differential energy loss of residues produced near the front or the back of the target. The theoretical momentum profiles must thus include this additional broadening. This effect was negligible for the two-neutron removal reaction and was strongest for the two-proton removal to ${ }^{36} \mathrm{Mg}$, when $\delta p=0.29 \mathrm{GeV} / c$.

Gamma-ray spectroscopy was performed in all of the measurements and allowed the identification of the residue final states: see Refs. [18,19,21] for details and $\gamma$-ray spectra. For the ${ }^{20,36} \mathrm{Mg}$ residues, the relative population of the $0^{+}$ground and $2^{+}$first excited states - the only states populated-were determined from the $\gamma$-ray yields in coincidence with the residues. These were used to adjust the proportions of the calculated $0^{+}$and $2^{+}$distributions when comparing to the measured inclusive momentum distributions. For the two-proton knockout to ${ }^{26} \mathrm{Ne}$, the first $4^{+}$state at $3.50 \mathrm{MeV}$ was directly populated and an exclusive momentum distribution of the ${ }^{26} \mathrm{Ne} 4_{1}^{+}$transition could be extracted using a software gate on the $4_{1}^{+} \rightarrow 2_{1}^{+}$ photopeak in the $\gamma$-ray spectrum.

Figure 4(a) compares the shapes of the measured and theoretical (solid curve) inclusive residue parallel momentum distribution from two-proton knockout of ${ }^{38} \mathrm{Si}$ at $83 \mathrm{MeV} / \mathrm{u}$. The $0^{+}$(dashed) and $2^{+}$(dotted) final states
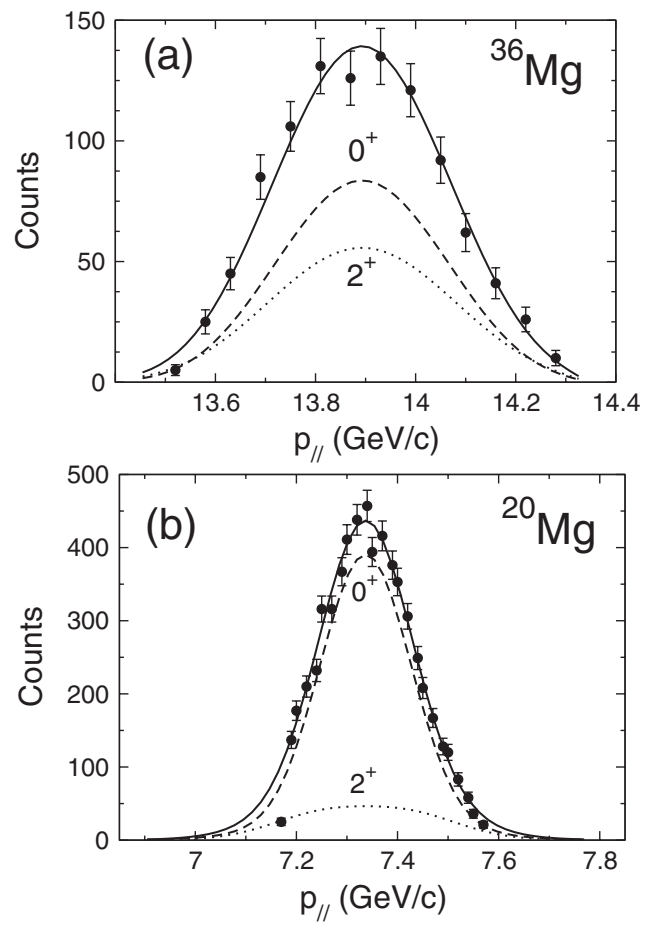

FIG. 4. Measured (symbols) and theoretical (solid curves) inclusive momentum distributions of (a) ${ }^{36} \mathrm{Mg}$ after two-proton knockout from ${ }^{38} \mathrm{Si}$ at $83 \mathrm{MeV} / \mathrm{u}$, and (b) ${ }^{20} \mathrm{Mg}$ after twoneutron knockout from ${ }^{22} \mathrm{Mg}$ at $75 \mathrm{MeV} / \mathrm{u}$. The two distributions are plotted over the same range of momenta so as to make clear their relative widths. The theoretical curves for the $0^{+}$ (dashed) and $2^{+}$(dotted) states are weighted according to the $\gamma$-ray data, being (a) $58 \%$ and $42 \%$ and (b) $84 \%$ and $16 \%$, respectively. All calculations take account of the beam-profile and differential momentum loss in the target. The latter is negligible in (b). 


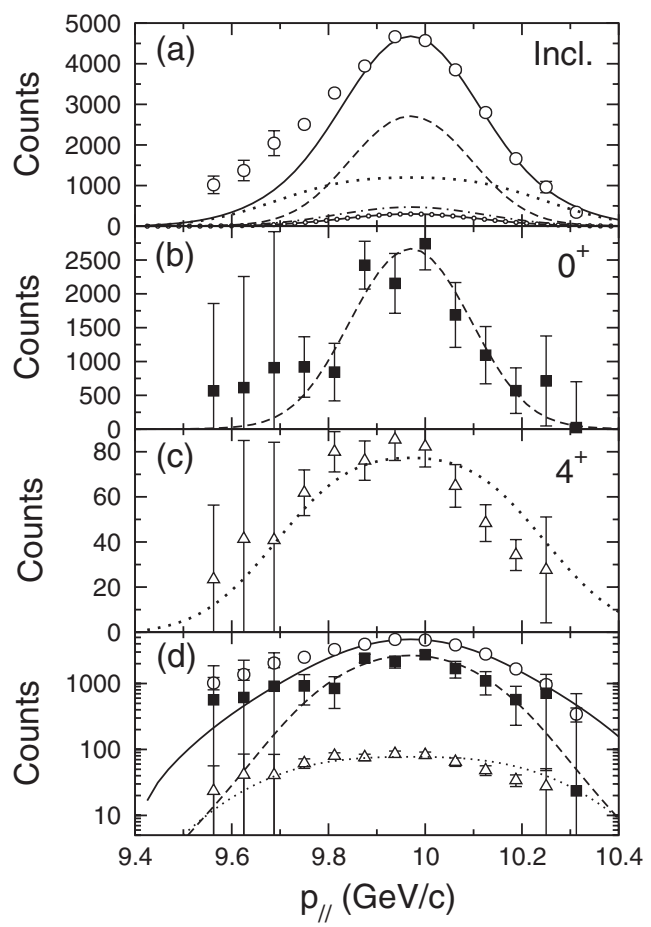

FIG. 5. Measured (symbols) and theoretical (curve) inclusive and exclusive parallel momentum distributions after two-proton knockout from ${ }^{28} \mathrm{Mg}$ at $82 \mathrm{MeV} / \mathrm{u}$. Panel (a) shows the measured inclusive distribution and that constructed from the weighted $0^{+}, 2_{1}^{+}, 4_{1}^{+}$and $2_{2}^{+}$distributions [13]. Panel (b) shows the ${ }^{26} \mathrm{Ne}\left(0^{+}\right.$, ground state) distribution, panel (c) the ${ }^{26} \mathrm{Ne}\left(4_{1}^{+}, 3.50 \mathrm{MeV}\right)$ final state distribution and panel (d) shows all distributions on a logarithmic scale. All the calculated shapes take account of the beam-profile $(0.1 \mathrm{GeV} / c)$ and differential energy loss in the target $(0.24 \mathrm{GeV} / c)$ broadening.

are weighted by the experimental yields from the $\gamma$-ray data, $58 \%$ and $42 \%$, respectively. The calculations have been folded with the beam-profile and the differential momentum loss in the target. The agreement with the data is excellent, however, as the beam momenta are restricted to $1.66 \%$, there is considerable broadening of the bare theoretical distributions, masking their markedly different widths.

Figure 4(b) shows the analogous results for two-neutron knockout from ${ }^{22} \mathrm{Mg}$ at $75 \mathrm{MeV} / \mathrm{u}$. Here the $0^{+}$and $2^{+}$ transitions have weights $84 \%$ and $16 \%$, respectively. Once again the agreement with the data is excellent. Since in this case the experimental broadening is rather minimal and the $0^{+}$transition is dominant, the very narrow (bare) shape of the $0^{+}$theoretical distribution is particularly evident.

The inclusive and partial cross sections for removal of two strongly bound protons from ${ }^{28} \mathrm{Mg}$ were analyzed previously [13,21]. When first reported [21], both the inclusive cross section and the shape of its parallel momentum distribution were described using a simplified, uncorrelated-nucleon knockout model. In that limit, the momentum distribution is independent of the final state $J$. The inclusive momentum distribution was reasonably described by this simple model, which predicts the convolution of the momentum distributions from two, independent $\pi\left[1 d_{5 / 2}\right]$ single-proton removals. In this work the data set has been reanalyzed to extract exclusive distributions for the $0^{+}$and $4_{1}^{+}$transitions. The measured shapes and the now fully correlated momentum distribution calculations are compared in Fig. 5. There is a significant differential energy loss broadening of the distributions and significant experimental uncertainties at the wings of the acceptance window. The overall agreement is good, but more detailed comparison will need a dedicated, improved-statistics, exclusive measurement.

In summary, we have formulated theoretical calculations for the final-state-exclusive parallel momentum distributions of projectile residues after direct two-nucleon knockout. The formalism was presented for the inelastic removal, or stripping mechanism; however, the calculations included the equally important diffraction plus stripping mechanism [13]. The calculations show that exclusive parallel momentum distribution measurements will have high spectroscopic value in determining the spins of states in very rare near-dripline nuclei. Applications of the methodology to three available measurements provided detailed confirmation of these theoretical expectations. Complete details of the formalism will be presented elsewhere [14].

This work was supported by the United Kingdom Science and Technology Facilities Council (STFC) under Grants No. ST/F012012 and EP/D003628, and the National Science Foundation under Grants No. PHY0606007, PHY-0555366 and PHY-0758099.

[1] J. A. Tostevin, J. Phys. G 25, 735 (1999).

[2] P. G. Hansen and J. A. Tostevin, Annu. Rev. Nucl. Part. Sci. 53, 219 (2003).

[3] B. A. Brown, P. G. Hansen, B. M. Sherrill, and J. A. Tostevin, Phys. Rev. C 65, 061601(R) (2002).

[4] A. Gade et al., Phys. Rev. C 69, 034311 (2004).

[5] A. Gade et al., Phys. Rev. Lett. 93, 042501 (2004).

[6] J. R. Terry et al., Phys. Rev. C 69, 054306 (2004).

[7] L. A. Riley et al., Phys. Rev. C 78, 011303(R) (2008).

[8] J. R. Terry et al., Phys. Lett. B 640, 86 (2006).

[9] A. Gade et al., Phys. Rev. C 71, 051301(R) (2005).

[10] A. Gade et al., Phys. Rev. C 74, 047302 (2006).

[11] T. Kobayashi et al., Phys. Rev. Lett. 60, 2599 (1988).

[12] J.A. Tostevin, G. Podolyák, B.A. Brown, and P.G. Hansen, Phys. Rev. C 70, 064602 (2004).

[13] J. A. Tostevin and B. A. Brown, Phys. Rev. C 74, 064604 (2006).

[14] E. C. Simpson and J. A. Tostevin (to be published).

[15] J. A. Tostevin, Eur. Phys. J. Special Topics 150, 67 (2007).

[16] B. A. Brown, Phys. Rev. C 58, 220 (1998).

[17] A. Gade et al., Phys. Rev. C 77, 044306 (2008).

[18] A. Gade et al., Phys. Rev. C 76, 024317 (2007).

[19] A. Gade et al., Phys. Rev. Lett. 99, 072502 (2007).

[20] D. Bazin et al., Nucl. Instrum. Methods Phys. Res., Sect. B 204, 629 (2003).

[21] D. Bazin et al., Phys. Rev. Lett. 91, 012501 (2003). 